\title{
A Descriptive Analysis of Prognostic Indicators in Patients with Non-Convulsive Status Epilepticus in a Tertiary Hospital Population
}

\author{
Chantelle Hrazdil, Raed Alroughani, Manouchehr Javidan
}

Neurophysiology Department and Epilepsy Program, Division of Neurology, Vancouver General Hospital, University of British Columbia, Vancouver, Canada.

Email: mjavidan@vch.ca

Received December $16^{\text {th }}, 2011$; revised January $25^{\text {th }}, 2012$; accepted February $15^{\text {th }}, 2012$

\begin{abstract}
Background: Non-convulsive status epilepticus (NCSE) is defined as a change in mental state of at least 30 minutes associated with continuous or nearly continuous epileptiform discharges. Identification of prognostic indicators can guide decision making surrounding the use of poorly established treatment interventions in this heterogeneous population. Methods: We identified 66 consecutive inpatients with NCSE. Data surrounding clinical, electrographic, and treatment factors were collected via a retrospective systematic review of medical records and electronic EEGs, and were correlated with discharge outcome (return to baseline, new disability, or death). Results: Of all subjects, $21 \%$ returned to baseline, $26 \%$ acquired new disability, and 53\% died, of whom half had anoxic encephalopathy. On univariate analysis, seventeen variables correlated significantly with death, although multivariate logistic regression analysis subsequently identified only comatose state and number of life threatening comorbidities as independent predictors of mortality. Of survivors, comatose state, critical care environment, length of hospital stay, and acute symptomatic seizures predicted new disability, with the latter two showing independent significance. Following exclusion of cases with anoxic encephalopathy, the use of an anaesthetic infusion was also an independent predictor of mortality. Conclusions: NCSE is associated with variable morbidity and mortality. While one fifth of our NCSE patients returned to baseline, those comatose with acute structural/metabolic seizures, anaesthetic infusions, and life threatening comorbidities were unlikely to survive without disability at discharge.
\end{abstract}

Keywords: Non-Convulsive Status Epilepticus; Morbidity; Mortality; Prognosis; Epilepsy; EEG

\section{Introduction}

There is variable nomenclature in the literature and no universally accepted definition of non-convulsive status epilepticus (NCSE). Most recognize NCSE as continuous or nearly continuous electrographic seizure activity associated with an alteration in mental state of at least 30 minutes, and occurring in the absence of frank convulsions [1-3]. The diagnosis is increasingly recognized with the advent of long term EEG monitoring and heightened physician awareness, although the incidence is still underestimated and constitutes at least one quarter of cases of status epilepticus $[4,5]$. The entity proves a diagnostic and therapeutic challenge given its pleomorphic clinical presentation, unclear urgency and required duration of EEG monitoring, lack of definitive electrographic criteria, and controversial treatment interventions [6,7]. Several chemical and electrical animal models of non-convulsive seizures provide a rational for the importance of timely diagnosis and treatment [8,9], although extrapolation to human NCSE remains difficult in the face of scarce clinical data, some of which raises concerns that our treatments may be worse than the disease [10-13].

Morbidity and mortality estimates for NCSE depend greatly on the epidemiology of the population studied, the definition of NCSE used, and seizure etiology [2]. In the hospital setting, NCSE tends to be associated with marked morbidity (39\%) and mortality (18\%) [14]. In fact, some studies have shown case fatalities upwards of fifty percent in NCSE in the critically ill or following convulsive status epilepticus (CSE) [11,15-17]. Although comorbid acute medical and neurological illnesses have been found to impact NCSE outcomes $[14,18,19]$, a recent case-control study showed that elderly patients with NCSE had a worse prognosis than age-matched controls with similar altered mental status and comorbidities [20].

In this study, we characterize NCSE demographics in an inpatient population, with special attention to differ- 
ences in etiology, mental state, EEG features, and treatment interventions. We endeavour to identify prognostic clinical and electrographic factors associated with morbidity and mortality to better guide clinical decision making, optimizing resource utilization and improving patient outcomes.

\section{Methods}

\subsection{Case Identification}

Following approval by our local ethics committee, we retrospectively reviewed the electronic EEG database at Vancouver General Hospital, University of British Columbia. All inpatient EEG requests with a question of non-convulsive seizures in patients with an unexplained decreased level of consciousness were studied over a five year period (May 14, 2003 through June 30, 2008). Patients were all 16 years of age or older and were located in critical care, emergency room, or acute and subacute hospital ward environments.

Similar to previous studies [21-23], a minimum 20 minute EEG recording was accepted, in lieu of the 30 minutes traditionally used to define status epilepticus (SE), given that routine EEGs are often of shorter duration and the clinical criterion of 30 minutes of altered mental state was always maintained. All EEGs had originally been read and reported by one of three board certified electroencephalographers. A cohort of possible NCSE cases was subsequently generated and original electronic EEGs were re-reviewed for evidence of NCSE. EEG technician and chart notes were supplemented with video footage, where available, to identify patient mental state and any subtle clinical signs correlating with suspicious electrographic ictal activity.

\subsection{Definition of NCSE}

Patients were identified according to our locally developed criteria for NCSE, as modified from previous papers and outlined in Table 1 [24-26]. All patients had an alteration in mental status lasting at least 30 minutes and occurring in the absence of frank convulsions. Electrographic seizure correlates may have entailed continuous epileptiform activity, a series of three or more subclinical seizures without intervening clinical improvement, or periodic epileptiform discharges with any one or more of the ictal features listed in Table 1. In an attempt to maintain clarity given their lack of specificity for an ictal state, isolated BiPLEDs (bilateral independent periodic lateralized epileptiform discharges) and GPEDs (generalized periodic epileptiform discharges) (unassociated with subtle abnormal movements) and triphasic waves were excluded. Those with pre-established epileptic encephalo- pathies, paralytic agents during their diagnostic recordings, and incomplete records were also excluded.

\subsection{Patient Data}

Patient charts were systematically reviewed for the following demographic variables: age, sex, hospital location, known epilepsy, preceding clinical seizures or SE, neuroimaging findings, metabolic derangements, life threatening medical comorbidities, and duration of hospital stay. Similar to Young et al. [24], seizures were classified as acute structural/metabolic if they occurred in the context of an acute brain insult or systemic disturbance in patients without pre-existing seizure disorders or brain lesions. The remainder included those with previously established seizure disorders or seizures from pre-existing non-progressive lesions, and were classified in accordance with the new International League Against Epilepsy (ILAE) criteria as remote structural/metabolic, genetic, or unknown [27].

\subsection{EEG Data}

The time from hospital admission to diagnostic EEG was recorded as was diagnostic EEG duration and the use of continuous EEG (CEEG), defined as long-term video monitoring over consecutive days. Level of consciousness was

Table 1. Diagnostic criteria for NCSE.

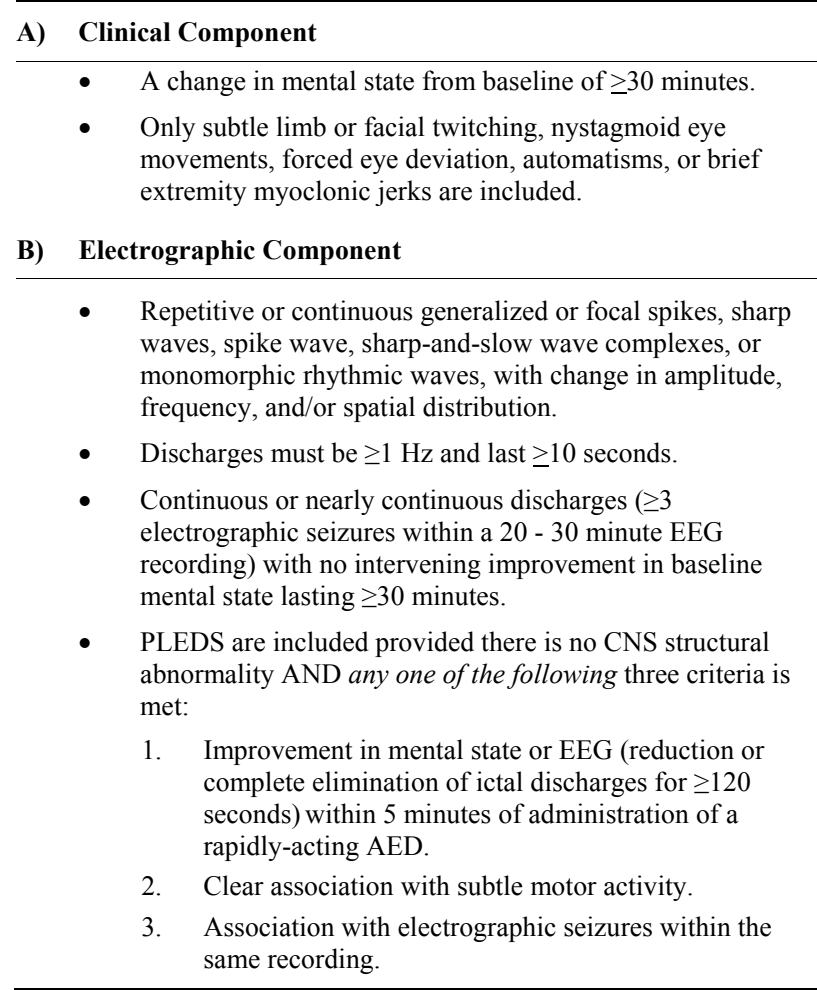

Periodic lateralized epileptiform discharges (PLEDS); Anti-epileptic drug (AED). Criteria modified from Young et al., 1996; Chong et al., 2005, Kaplan et al., 2007. 
documented as comatose or not. Subtle motor activity at the time of EEG diagnosis was noted. Patients were categorized based on electrographic criteria at NCSE diagnosis: 1) continuous epileptiform activity for $\geq 20$ minutes; 2 ) $\geq$ three subclinical seizures within 20 - 30 minutes of recording; or 3) PLEDS with ictal features (see Table 1). Electrographic discharges were further characterized as focal or generalized. When available, serial EEGs were reviewed and the time of electrographic seizure cessation was observed.

\subsection{Treatment Initiation}

Individual antiepileptic drugs (AEDs) used were recorded sequentially and divided into those used pre and postdiagnostic EEG interventions. The time from definitive diagnosis of NCSE to subsequent treatment was noted and characterized as over or under one hour. Any ensuing use of IV anaesthetic infusions was documented.

\subsection{Treatment Response}

Immediate electrographic response to a rapidly acting AED, usually a benzodiazepine, was recorded where available, and categorized as partial (reduction in discharge frequency or complete elimination of epileptiform activity lasting under five minutes) vs complete (persistent elimination of epileptiform activity for the remainder of the recording). Patients were also observed for an associated clinical improvement. Patients were defined as treatment refractory if they failed to show a clinical or electrographic response to two first line AEDs.

\subsection{Outcome Measures}

Discharge outcome served as our primary outcome measure and was defined as: 1) return to baseline, 2) new disability, or 3) death. For those who survived, discharge disposition was categorized as: 1) return to previous home environment, or 2) discharge to a subacute, rehabilitation, or long term care facility.

\subsection{Statistics}

Data were analyzed using the SAS software package. We conducted a univariate analysis to identify significant associations with mortality in the entire cohort and morbidity amongst survivors. Categorical variables were compared by Chi square or Fisher exact tests as needed, whereas Student's t-test and Mann-Whitney U tests were applied for normally distributed and non-normally distributed continuous variables respectively. Highly correlated variables included admission diagnosis of cardiac arrest, and respiratory and cardiac comorbidities, thus these were omitted. The remainder of the statistically significant variables $(p<0.05)$ were included in a stepwise multivariate logis- tic regression analysis to identify those independently associated with mortality in all patients and new disability amongst survivors. The statistical analysis was later repeated with exclusion of all anoxic cases.

\section{Results}

\subsection{Prevalence of NCSE}

A total of 1460 patients were screened, 66 of whom met our diagnostic criteria for NCSE, giving a period prevalence of $4.5 \%$.

\subsection{Clinical Characteristics of the Study Group}

Mean age at onset of NCSE was 58 years (range 18 - 90); $38 \%$ were female. Twenty percent $(n=13)$ had a known diagnosis of epilepsy. Preceding overt clinical seizures were present in $67 \%(n=44)$, about half of whom had generalized, convulsive, partial, or myoclonic SE $(n=20)$. Eighty percent of the NCSE cohort $(n=53)$ had acute structural/metabolic seizures. Table 2 illustrates the primary

Table 2. Mortality and morbidity according to primary NCSE etiology.

\begin{tabular}{cccc}
\hline Etiology & $\begin{array}{c}\text { Number } \\
\text { (\%) of } \\
\text { Patients }\end{array}$ & $\begin{array}{c}\text { Number } \\
\text { (\%) of } \\
\text { Deaths }\end{array}$ & $\begin{array}{c}\text { Number } \\
\text { (\%) of } \\
\text { Newly Disabled }\end{array}$ \\
\hline $\begin{array}{c}\text { Acute } \\
\text { Structural/Metabolic }\end{array}$ & $53(80)$ & $35(100)$ & $13(76)$ \\
HA encephalopathy & 20 & 19 & 1 \\
ICH/head trauma & 7 & 4 & 3 \\
Uncertain & 7 & 4 & 2 \\
Ischemic stroke & 4 & 2 & 2 \\
Substance W/D & 3 & 0 & 2 \\
Systemic illness & 3 & 1 & 1 \\
CNS infection & 4 & 3 & 0 \\
Post-intracranial surgery & 2 & 1 & 0 \\
PRES & 1 & 0 & 1 \\
Brain tumor & 1 & 0 & 1 \\
Dural AV fistula & 1 & 1 & 0 \\
Remote & $10(15)$ & $0(0)$ & $4(24)$ \\
Structural/Metabolic & 4 & 0 & 1 \\
Previous cerebral infarction & 4 & 0 & 2 \\
Remote head trauma & 3 & 0 & 0 \\
Known vascular & 2 & 0 & 1 \\
malformation & 1 & $0(0)$ & $0(0)$ \\
Cerebral palsy & $2(2)$ & $0(0)$ & $0(0)$ \\
Genetic & $1(3)$ & 35 & 17 \\
Unknown & 66 & & \\
TOTAL & & & \\
\hline
\end{tabular}

HA: hypoxic-anoxic encephalopathy; Substance W/D: alcohol or benzodiazepine withdrawal. 
etiology of NCSE in more detail. The majority of patients were critically ill with $68 \%(\mathrm{n}=45)$ requiring intensive care at the time of their diagnostic EEG. In fact, life threatening comorbidities were present in $85 \%$ of subjects $(\mathrm{n}=$ 56), with details as demonstrated in Table 3 . All but one patient underwent neuroimaging, of whom $74 \%(n=48)$ had cerebral structural pathology.

\subsection{EEG Features}

The median time from hospital admission to diagnostic EEG was 2.5 days. EEG durations ranged from $20 \mathrm{~min}-$ utes to several days of CEEG monitoring, with a mean recording of 75 minutes at the time of diagnosis. CEEG monitoring was employed in $48 \%$ of cases $(n=32)$, the implementation of which depended on the judgment of the treating epileptologist, and was often influenced by the clinical context and initial EEG findings. Aside from those post-cardiac arrest, patients were monitored with CEEG after EEGs demonstrating subclinical continuous electrographic seizures in all but one case (92\%). CEEG was also far more commonly used for those comatose $(71 \%)$, critically ill in ICU (70\%), and on anaesthetic infusions $(89 \%)$. Those non-comatose on the wards with a series of subclinical seizures or periodic epileptiform discharges often had serial portable EEGs of varying durations as a substitute.

Table 3. Significant predictors of mortality and morbidity in NCSE on univariate analysis.

\begin{tabular}{|c|c|c|c|c|c|}
\hline & \multirow[t]{2}{*}{ Dead } & \multicolumn{2}{|c|}{ Alive } & \multirow[b]{2}{*}{ (I vs II \& III) } & \multirow[b]{2}{*}{ (II vs III) } \\
\hline & & New disability & Baseline & & \\
\hline Variables & $\mathrm{I}(\mathrm{n}=35)$ & II $(\mathrm{n}=17)$ & III $(n=14)$ & p mortality^ & p morbidity ${ }^{\#}$ \\
\hline \multicolumn{6}{|l|}{ Baseline characteristics } \\
\hline *Prior diagnosis of epilepsy, $\mathrm{n}(\%)$ & $1(3)$ & $5(29)$ & $7(50)$ & 0.0003 & 0.241 \\
\hline Admitting diagnosis of cardiac arrest, $\mathrm{n}(\%)$ & $17(26)$ & 0 & 0 & $<0.0001$ & . \\
\hline \multicolumn{6}{|l|}{ Hospital resource utilization } \\
\hline Hospital days (median, range) & $8(0-134)$ & $48(5-136)$ & $12(4-57)$ & 0.004 & 0.0003 \\
\hline *Critical care environment during diagnostic EEG, $\mathrm{n}(\%)$ & $32(91)$ & $11(65)$ & $2(14)$ & $<0.0001$ & 0.0094 \\
\hline *Use of CEEG monitoring, $\mathrm{n}(\%)$ & $23(66)$ & $6(35)$ & $3(21)$ & 0.0029 & 0.4564 \\
\hline \multicolumn{6}{|l|}{ Primary cause of NCSE } \\
\hline *Acute structural/metabolic seizures, $\mathrm{n}(\%)$ & $35(100)$ & $13(76)$ & $5(36)$ & $<0.0001$ & 0.0325 \\
\hline Hypoxic-anoxic etiology & $19(54)$ & $1(6)$ & $0(0)$ & $<0.0001$ & 1 \\
\hline \multicolumn{6}{|l|}{ Characteristics of NCSE } \\
\hline${ }^{*}$ Comatose during diagnostic EEG, $\mathrm{n}(\%)$ & $34(97)$ & $7(41)$ & $1(7)$ & $<0.0001$ & 0.0454 \\
\hline Generalized EEG pattern, n (\%) & $23(66)$ & $2(12)$ & $2(14)$ & $<0.0001$ & 1 \\
\hline Continuous electrographic epileptiform activity & $19(54)$ & $5(29)$ & $1(7)$ & 0.0035 & 0.1848 \\
\hline \multicolumn{6}{|l|}{ Treatment } \\
\hline *Treatment refractory, n (\%) & $35(100)$ & $10(67)$ & $7(54)$ & $<0.0001$ & 0.4885 \\
\hline *Use of anaesthetic infusion for seizure control, $\mathrm{n}(\%)$ & $27(77)$ & $4(24)$ & 0 & $<0.0001$ & 0.1075 \\
\hline *Number of AEDs trialed, mean & 3.7 & 3.7 & 2.5 & 0.3548 & 0.2100 \\
\hline \multicolumn{6}{|l|}{ Hospital complications } \\
\hline *Mean number of life threatening comorbidities & 2.5 & 1.2 & 0.9 & $<0.0001$ & 0.3595 \\
\hline *Respiratory compromise, $\mathrm{n}(\%)$ & $35(100)$ & $8(47)$ & $3(23)$ & $<0.0001$ & 0.1328 \\
\hline *Cardiac comorbidity, n (\%) & $20(57)$ & 0 & 0 & $<0.0001$ & . \\
\hline *Shock, n (\%) & $22(63)$ & $5(29)$ & $4(29)$ & 0.0055 & 0.9598 \\
\hline *Mean number of metabolic derangements & 1.7 & 1.1 & 0.7 & 0.0037 & 0.846 \\
\hline
\end{tabular}

Boldface indicates $\mathrm{p}$ value $<0.05$; ${ }^{\wedge}$ Compares fatal with nonfatal NCSE; ${ }^{\#}$ Compares survivors with residual disability to those who returned to baseline. *Variables associated with mortality after exclusion of cases of anoxic encephalopathy. 
Sixty four percent of the cohort $(n=42)$ was comatose at the time of diagnostic EEG. Subtle motor activity, such as blinking, facial or low amplitude limb twitching, forced eye deviation, or nystagmoid eye movements, was noted in $64 \%$ of cases $(n=42)$. Figures 1 and 2 illustrate examples of EEGs diagnostic of NCSE. Table 4 summa-

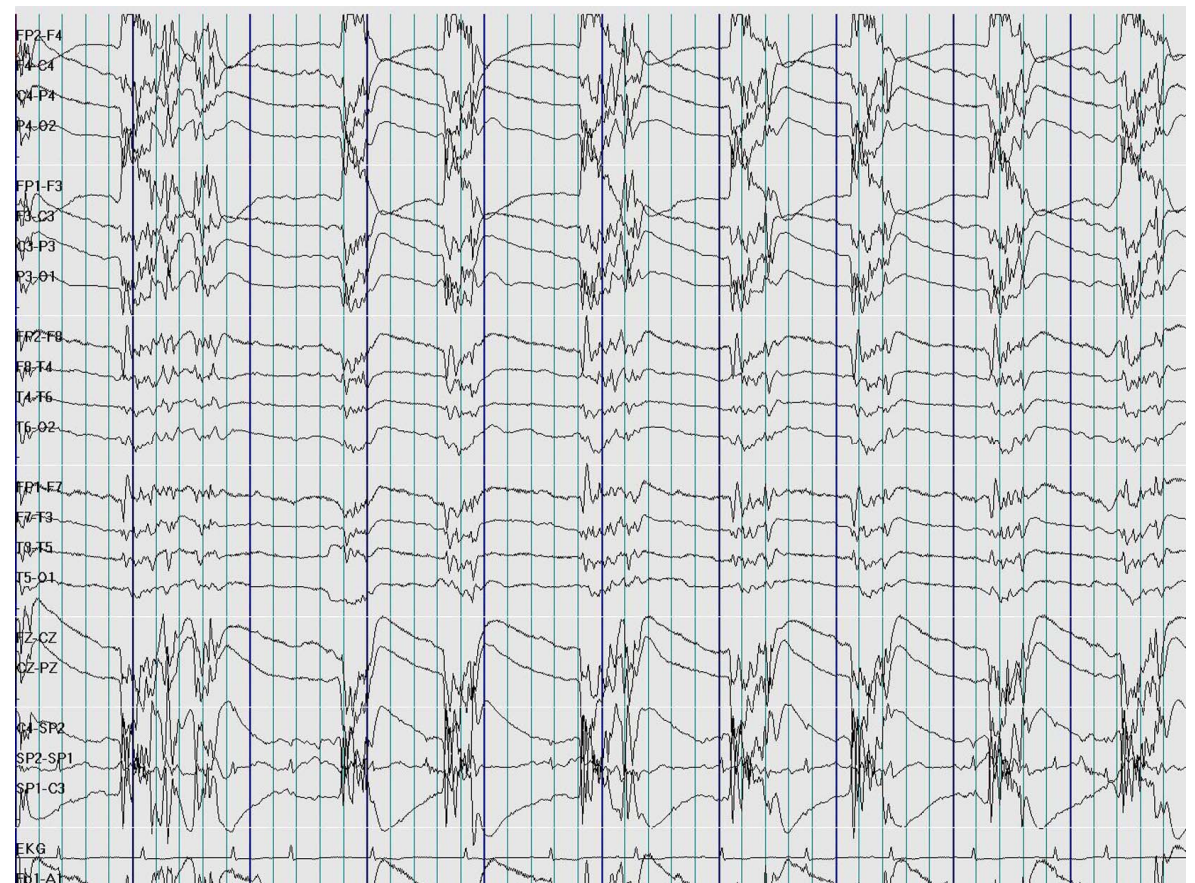

Figure 1. Example of EEG NSCE criteria A): A 68 year old male was admitted to ICU in a coma following a cardiac arrest. Imaging confirmed a hypoxic-anoxic brain injury. CEEG demonstrated continuous generalized predominantly frontal central polyspike activity at $0.5-1 \mathrm{~Hz}$. The only motor activity was occasional slight chin twitches and upward eye movements.

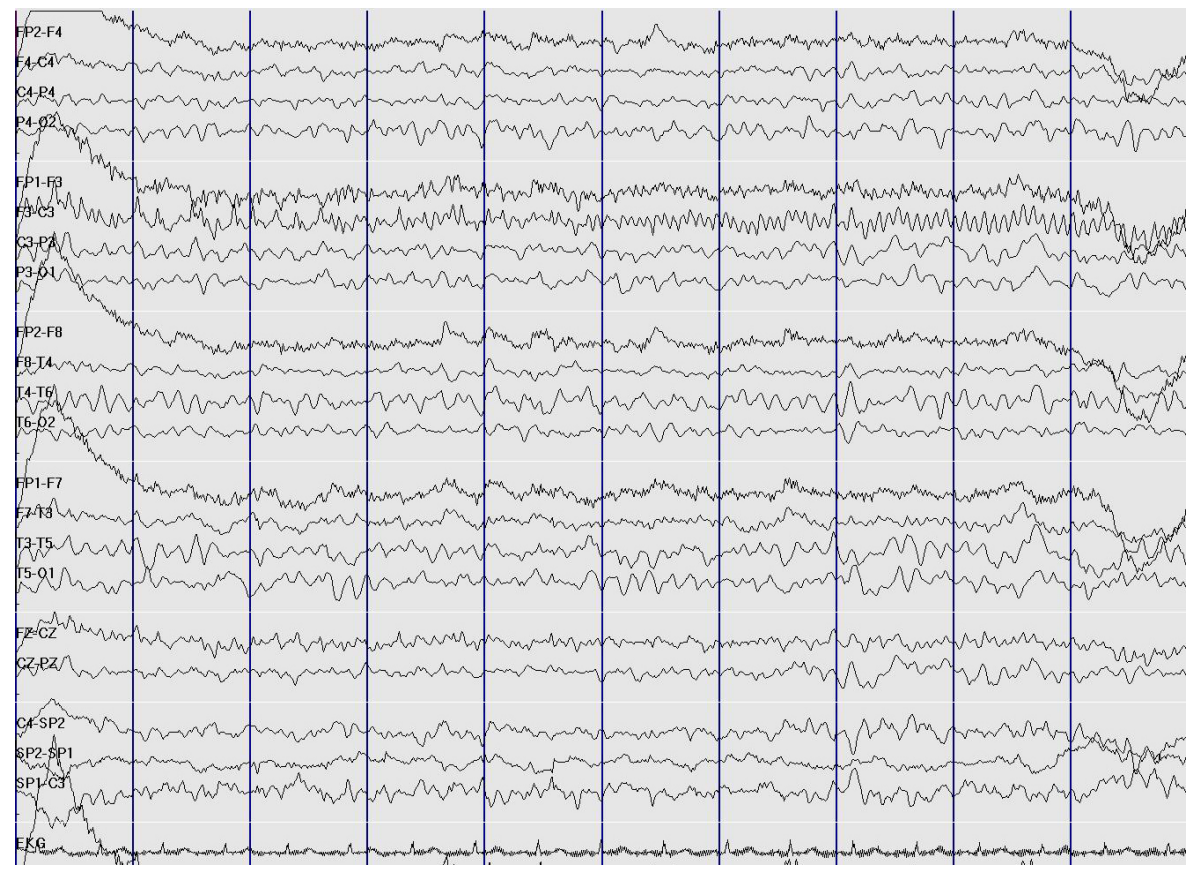

Figure 2. Example of EEG NCSE criteria B): A 34 year old woman with known epilepsy due to an old left frontal stroke presented with right focal motor seizures and fluctuating confusion. Her EEG demonstrated a build-up of rhythmic $10-11 \mathrm{~Hz}$ activity over the left frontal electrodes (F3 and FP1). There was associated expressive dysphasia without any motor activity. She experienced four subclinical seizures within 30 minutes. 
Table 4. Outcomes according to EEG characteristics at NCSE diagnosis.

\begin{tabular}{lcccc}
\hline & $\begin{array}{c}\text { All } \\
\text { Cases }\end{array}$ & Dead & $\begin{array}{c}\text { New } \\
\text { Disability }\end{array}$ & $\begin{array}{c}\text { Return to } \\
\text { Baseline }\end{array}$ \\
\hline Variables, n (\%) & 66 & $35(53)$ & $17(26)$ & $14(21)$ \\
EEG NCSE criteria & & & & \\
$\begin{array}{l}\text { A) Continuous } \\
\text { epileptiform activity }\end{array}$ & $25(38)$ & $19(54)$ & $5(29)$ & $1(7)$ \\
$\begin{array}{l}\text { B) At least three subclinical } \\
\text { seizures in 20 - 30 mins }\end{array}$ & $35(53)$ & $15(43)$ & $11(65)$ & $9(64)$ \\
$\begin{array}{l}\text { C) PLEDs with ictal } \\
\text { features (see Table 1) }\end{array}$ & $6(9)$ & $1(3)$ & $1(6)$ & $4(29)$ \\
$\begin{array}{l}\text { EEG NCSE focality } \\
\begin{array}{l}\text { Generalized } \\
\text { electrographic seizures }\end{array}\end{array}$ & $27(41)$ & $23(66)$ & $2(12)$ & $2(14)$ \\
$\begin{array}{l}\text { Focal } \\
\text { electrographic seizures }\end{array}$ & $39(59)$ & $12(34)$ & $15(88)$ & $12(86)$ \\
\hline
\end{tabular}

rizes some key EEG features. Over half of patients ( $\mathrm{n}=$ $35,53 \%$ ) met NCSE diagnostic criteria B) through the presence of a series of subclinical seizures, $38 \%(n=25)$ were found to have continuous epileptiform activity A), and the remaining $9 \%(\mathrm{n}=6)$ displayed PLEDs with ictal correlates C), $66 \%$ of whom had associated subclinical seizures in the same recording. Focal epileptiform activeity $(\mathrm{n}=39,59 \%)$ was more common than generalized patterns $(\mathrm{n}=27,41 \%)$.

Of the fifty two patients $(79 \%)$ who underwent repeat EEGs, $62 \%(\mathrm{n}=32)$ achieved electrographic seizure cessation, while $35 \%(\mathrm{n}=18)$, all of whom died, had ongoing electrographic seizure activity at the time of their last study.

\subsection{Treatment Initiation}

Ninety one percent of patients $(n=60)$ received at least one AED, often a benzodiazepine, prior to their diagnostic EEG. A mean of two AEDs (range 0 - 5) had been initiated prior to NCSE diagnosis. Ninety one percent of subjects $(\mathrm{n}=60)$ received additional AED management upon diagnosis of NCSE. Fifty two percent of these patients $(\mathrm{n}=31)$ also received an anaesthetic infusion, most commonly midazolam, followed by propofol, thiopental, and rarely ketamine. NCSE treatment was delayed by over one hour in $53 \%$ of cases $(n=32)$. A mean of 3.5 AEDs were used per patient (range 1 - 10).

\subsection{Treatment Response and Outcome}

Electrographic response to AEDs was studied in 35 cases, of whom four (11.4\%) showed a complete electrographic response, twenty six $(74.3 \%)$ showed a partial electrographic improvement, and five (14.3\%) showed no electrographic change. Only four patients (11.4\%) demonstrated an associated improvement in mental state. Eighty three percent $(n=52)$ of the 63 treated patients were refractory to two or more first line AEDs. Table 5 demonstrates our primary outcome measure with a mortality rate of $53 \%(\mathrm{n}=35)$ and $26 \%(\mathrm{n}=17)$ accrual of new disability. Of those who survived $(\mathrm{n}=31), 61 \%(\mathrm{n}=19)$ were discharged to their previous home environments, with the remainder being transferred to a subacute, rehabilitation, or long term care facility. The median hospital stay was 13 days (range 1 - 136).

\subsection{Predictors of Mortality}

Mortality varied greatly depending on primary NCSE etiology (Table 2), ranging from $0 \%$ in those with predating genetic or unknown epilepsies to $100 \%$ in those admitted with cardiac arrest. Univariate analysis identified seventeen variables that were associated with mortality (Table 3). However, following multivariate logistic regression analysis, only two factors were found to be significant independent predictors of death: 1) comatose state, and 2) number of life threatening comorbidities.

The data was re-analyzed upon exclusion of our twenty patients with hypoxic-anoxic encephalopathy, $95 \%$ of who died. Significant predictors of mortality on univariate analysis were unchanged aside from the addition of the number of AEDs used $(p=0.0316)$ and the presence of shock $(p=0.0502)$ as predictors of death. Admitting diagnosis of cardiac arrest, hypoxic-anoxic etiology, duration of hospital stay, generalized EEG pattern, and continuous epileptiform activity were no longer correlated with mortality. The subsequent multivariate logistic regression analysis identified use of an anaesthetic infusion and number of life-threatening comorbidities as independent predictors of death.

\subsection{Predictors of Functional Disability}

Amongst survivors, univariate analysis identified four variables associated with functional disability (Table 3): 1) comatose state, 2) critical care environment during diagnostic EEG, 3) longer length of hospital stay, and 4)

Table 5. NCSE primary outcome measure.

\begin{tabular}{ccc}
\hline Discharge outcome & $\begin{array}{c}\text { Number of } \\
\text { patients (n) }\end{array}$ & $\begin{array}{c}\text { Percentage of } \\
\text { patients (\%) }\end{array}$ \\
\hline Return to baseline & 14 & 21 \\
New disability & 17 & 26 \\
Dead & 35 & 53 \\
\hline
\end{tabular}


acute structural/metabolic seizures. The last two factors were subsequently isolated as significant independent predictors of new disability at hospital discharge on multivariate logistic regression analysis. These results were unchanged when the statistical analysis was repeated following exclusion of the hypoxic-anoxic cases.

Age, preceding clinical SE, treatment delay over 1 hour, and the total number of AEDs did not significantly correlate with death or disability on univariate analysis, nor did these variables alter the end results when rerun in the logistic regression analysis.

\section{Discussion}

The literature on NCSE is confounded by inconsistencies in its definition. The criteria for electrographic seizure established by Young et al. in 1996 are probably the best available, however with caveats and subsequent propositions for modification [24-26]. Specifications surrounding a significant improvement in clinical state or baseline EEG after an AED are left unclear. Our study corroborates the work of Drislane et al. in that many patients showed only a non-specific transient or partial electrographic response to IV AED administration in the face of comorbid structural CNS pathology or alternate etiologies for encephalopathy [22-45]. Only $11 \%$ of our tested patients showed an immediate clinical response to AEDs. This value is even lower than the $39 \%$ found by Shneker et al, who concluded that a response to benzodiazepines is not required to make a diagnosis of NCSE [14]. We thus elected to remove treatment response from our NCSE definition, aside from its role in potentially helping to define PLEDS as ictal [25]. Interestingly, morbidity (17\%) and mortality (17\%) were comparatively lower in our periodic epileptiform discharge subgroup, suggesting a more benign course in this population.

The rate of occurrence of NCSE in our tertiary hospital population with altered mental state and clinician suspicion of subclinical seizures is $4.5 \%$. This compares with numbers ranging from $1.2 \%$ to $37 \%$, depending not only on the NCSE definition applied, but also on the population studied, rigour of screening, and intensity of EEG monitoring $[23,28-33]$. This period prevalence is almost certainly an underestimate given this study's with unexplained alteration in mental state, and restricted use of CEEG monitoring.

In the majority of patients, clinicians were alerted to consider a diagnosis of NCSE given preceding clinical seizures (67\%) and/or subtle motor signs (64\%) during the diagnostic EEG. Yet $27 \%$ of diagnoses $(n=18)$ would have been missed using these clinical correlates alone. This illustrates the ongoing importance of a clinical index of suspicion for NCSE in patients with an unexplained alteration in consciousness, even in the absence of predating seizures or subtle motor features.

A subset of NCSE patients do well, as evidenced by one fifth of our population returning to their neurologic baseline at discharge. We've again shown that a history of epilepsy is a favourable prognostic indicator [11,34], as our low mortality rate of $8 \%$ in this population compares well with that of $2 \%-3 \%$ found by others $[14,35]$. In fact, our single death in a patient with a known seizure disorder was clearly not epilepsy-related as it occurred in the context of a cardiac arrest with postanoxic NCSE.

Despite the general good outcomes in those with known epilepsy, our overall mortality rate of $53 \%$ and $26 \%$ acquisition of new disability mirrors the work of Young et al. [24], indicating that inpatient NCSE needs to be addressed seriously. Indeed, the majority of our study population sustained at least one concurrent life-threatening illness $(85 \%)$, with many requiring critical care resources $(68 \%)$. Intuitively and in accordance with previous research $[11,14,20]$, we found that the number of life threatening comorbidities is independently associated with mortality $(\mathrm{p}<0.0001)$. And while comorbidities often predate or contribute to the cause of NCSE, others are clearly the sequelae of refractory seizures, aggressive therapeutic interventions, and long hospital stays.

The study of postanoxic NCSE exemplifies that etioly weighs heavily on outcome. We've demonstrated a $100 \%$ mortality rate in NCSE post-cardiac arrest, independent of various treatment efforts such as hypothermia (39\%) and IV anaesthetic infusions (72\%). Others have also shown that patients with NCSE following cerebral anoxia almost invariably have a fatal outcome [36-39]. The same holds true for postanoxic generalized myoclonus, despite the successful use of propofol infusions to control elecographic seizure activity [40]. One group has recently prosed that postanoxic NCSE be used as an independent poor prognostic indicator in cardiac arrest survivors [41]. Our study mortality rate dropped to $35 \%$ with exclusion of our twenty patients with hypoxic-anoxic encephalothy.

Studies of SE have established that prognosis is also greatly influenced by level of consciousness at presentation $[14,20,22,30,42-44]$. Not only are the causes of coma often fatal, but decreased level of consciousness predisposes to complications, such as respiratory compromise. Extrapolation to NCSE was demonstrated in a recent study [14], and is further supported by our identification of comatose state as an independent predictor of mortality $(\mathrm{p}<0.0001)$. Also in conjunction with the work of Shneker et al., we found a mortality rate of only 3\% amongst those who were non-comatose at NCSE diagnosis [14].

Persistent NCSE after the control of CSE (subtle SE) has been shown to have a mortality of $50 \%-65 \%$, with an additional $8 \%$ experiencing new or increased depend- 
ency $[16,17]$. We have replicated this data with a synonymous $50 \%$ mortality in our cases of subtle SE, and higher $30 \%$ acquisition of new disability. Interestingly however, preceding clinical SE did not significantly impact outcome in our study, illustrating the stronger contributions of other factors.

Consistent with a previous detailed electrographic analysis of NCSE performed by Granner et al., we categorized patients as having continuous epileptiform activity if they had continuous or persistent discharges, with brief breaks lasting no more than a few seconds [46]. We also included patients with repeated electrographic seizures and no intervening improvement in mental state, as these met the commonly accepted definition of SE. Not surprisingly, those with continuous epileptiform activity demonstrated worse outcomes than those with repeated subclinical seizures, with mortality rates of $76 \%$ vs $43 \%$ respectively. This suggests that persistent electrographic abnormalities either reflect more devastating underlying seizure etiologies, or contribute to accumulating neurologic disability. Perhaps the former is most likely given that this relationship did not persist upon exclusion of hypoxic-anoxic encephalopathy, a group in which both continuous epileptiform activity and mortality was ubiquitous.

Also of interest is the relationship between the focality of epileptiform discharges and outcome. Drislane et al. studied a series of patients with generalized electrographic $\mathrm{SE}$ in the context of severe intercurrent medical illness [22]. They found an $88 \%$ mortality rate, comparing well with our $85 \%$ mortality amongst generalized NCSE patients. This alarming number is likely explained by the fact that both studies included patients with postanoxic SE, all of whom had generalized electrographic ictal patterns. To the contrary, a recent study of NCSE failed to show a relationship between generalized spike wave discharges and outcome, likely because it did not include patients with acute anoxic brain injuries [14]. Likewise, this relationship did not persist in our cohort upon elimination of postanoxic cases.

Eighty percent of patients in our cohort experienced acute structural/metabolic seizures. This variable, in addition to length of hospital stay, has previously been shown to be an independent predictor of functional disability in all comers with SE [47]. We have, for the first time, proven the utility of this information for the same purposes amongst survivors with NCSE $(p<0.0001$ and $p=0.0010$ respectively). A longer hospital stay is undoubtedly related to the number and severity or medical comorbidities, although a component may be due to additional disability acquired due to non-convulsive seizures.

Because of the general lack of prospective randomized controlled trials in this challenging and difficult to study population, the optimal treatment of NCSE remains un- known. The only such study to our knowledge looked at 134 patients with subtle generalized CSE and found that only $15 \%$ showed a sustained electrographic and clinical response to their first AED, independent of the agent used [16]. Similarly, only $17 \%$ of our NCSE population responded to two or fewer AEDs. This treatment refracttory nature of NCSE is often felt to be a marker of more severe underlying neurologic disease, but also likely reflects a delay in its diagnosis and management.

NCSE is often present for hours to days before diagnosis, with seizures of longer duration being more difficult to treat [24]. However we've corroborated the SE work of Rossetti et al. [42], as we were unable to find a significant difference in outcomes amongst patients treated under vs over one hour from EEG diagnosis. While this is in part due to frequent AED initiation prior to NCSE confirmation, a lack of timing of treatment effect may also be explained by the heavier influence of underlying seizure etiology and comorbidities in a heterogeneous population such as ours.

One study of NCSE in the critically ill elderly found that the use of IV benzodiazepines was associated with increased adverse outcomes and mortality compared to those managed less aggressively [11]. At our institution, almost all patients had been treated with some form of AED prior to their formal diagnosis of NCSE, with just over $50 \%$ receiving anaesthetic infusions for control of subsequently confirmed NCSE. While the use of an infusion carries risks and was independently correlated with mortality on our multivariate analysis of non-anoxic cases, this does not establish cause and effect. Rather, the infusion group largely consisted of treatment refractory patients with severe underlying systemic or neurologic insults who were predestined to do poorly. Postanoxic NCSE aside, we believe that timely and individualized treatment of NCSE can positively impact outcome, as our interventions contributed to a $66 \%$ survival rate amongst nonanoxic cases, with two thirds of these returning to their home environments at discharge. In terms of NCSE postcardiac arrest, while an EEG may help guide prognosis [41], the balance of evidence argues against aggressive antiepileptic treatment in this cohort.

Our study has limitations given its unblinded retrospective nature and short follow up. Furthermore, CEEG monitoring was used in only one half of cases, thus eliminating our ability to consistently establish NCSE duration and electrographic response to treatment. This situation does however best replicate clinical practice in many institutions, where access to emergency CEEG is restricted by resource availability. Recognizing that there are flaws to incorporating such a diverse group of conditions under the unifying diagnosis of NCSE, we've studied the entire spectrum of disease in order to characterize the variabil- 
ity encountered in clinical practice and reflected differences in treatment choices and ultimate outcomes. To that end, the generalizability of our data is limited to patients admitted to a tertiary care hospital with access to EEG monitoring, deemed of diagnostic importance in the context of an unexplained altered mental state.

Lastly, our statistical power is limited by a small sample size. Yet there have been no large controlled studies of NCSE, with most of the literature centering on small case series $[10,11,21,24,28,31,32,35,48,49]$ and a case control study of 19 elderly [20]. Aside from a single paper assessing morbidity and mortality in 100 patients with NCSE [14], our series is the largest descriptive study of NCSE to date and the only one to systematically correlate NCSE clinical and electrographic variables with functional disability at discharge.

\section{Conclusions}

Our study shows that NCSE mortality rates are higher in those comatose with life threatening medical comorbidities and the need for an anaesthetic infusion, particularly those with hypoxic-anoxic encephalopathy. To the contrary, patients with known epilepsy who present with a milder impairment in mental status often do well. This paper thus complements the existing literature, illustrating the heterogeneity of NCSE and the several uncertainties surrounding its diagnosis and management. Given that NCSE adverse outcomes are invariably related to underlying etiology or subsequent complications, it remains difficult to prove the likely contribution of prolonged electrographic seizures. Yet a strong rational for prompt and individualized NCSE treatment persists.

While refractory NCSE has been reported to respond to midazolam, propofol or pentobarbital, randomized controlled trials of treatments for NCSE are lacking. We highlight the need for larger prospective studies into the natural history of the various subtypes of NCSE and a risk benefit analysis of its different treatment regimens. In the interval and while we continue to wait for evidence and resources to support widespread use of CEEG monitoring in the screening and management of NCSE, we recommend that, as a minimum, an EEG should be ordered on any patient with an unexplained altered mental state and critical illness requiring ICU care, coma, predating clinical seizures or status epilepticus, subtle suspicious motor activity, and/or known epilepsy.

\section{Acknowledgements}

The authors thank Helene Chou for her work performing EEG screening, Larry Stevenson for his help with the EEG database, and Jonathan Money and Karel Hrazdil for their assistance with the statistical analysis.

\section{REFERENCES}

[1] J. N. Gaitanis and F. W. Drislane, "Status Epilepticus: A Review of Different Syndromes, Their Current Evaluation, and Treatment," Neurologist, Vol. 9, No. 2, 2003, pp. 61-76. doi:10.1097/01.nrl.0000051445.03160.2e

[2] P. W. Kaplan, "The Clinical Features, Diagnosis, and ProGnosis of Nonconvulsive Status Epilepticus," Neurologist, Vol. 11, No. 6, 2005, pp. 348-361. doi:10.1097/01.nrl.0000162954.76053.d2

[3] S. Riggio, "Nonconvulsive Status Epilepticus: Clinical Features and Diagnostic Challenge," Psychiatric Clinics of North America, Vol. 28, No. 3, 2005, pp. 653-664.

[4] M. Walker, H. Cross, S. Smith, et al., "Nonconvulsive Status Epilepticus: Epilepsy Research Foundation Workshop Reports," Epileptic Disorders, Vol. 7, No. 3, 2005, pp. 253-296.

[5] F. Rosenow, H. M. Hamer and S. Knake, "The Epidemiology of Convulsive and Nonconvulsive Status Epilepticus," Epilepsia, Vol. 48, Suppl. 8, 2007, pp. 82-84. doi:10.1111/j.1528-1167.2007.01359.x

[6] R. Maganti, P. Gerber, C. Drees and S. Chung, "Nonconvulsive Status Epilepticus," Epilepsy \& Behavior, Vol. 12, No. 4, 2008, pp. 572-586. doi:10.1016/j.yebeh.2007.12.002

[7] H. Meierkord and M. Holtkamp, "Non-Convulsive Status Epilepticus in Adults: Clinical Forms and Treatment," Lancet Neurology, Vol. 6, No. 4, 2007, pp. 329-339. doi:10.1016/S1474-4422(07)70074-1

[8] E. Lothman, "The Biochemical Basis and Pathophysiology of Status Epilepticus," Neurology, Vol. 40, Suppl. 2, 1990, pp. 13-23.

[9] B. S. Meldrum, R. A. Vigouroux and J. B. Brierley, "Systemic Factors and Epileptic Brain Damage. Prolonged Seizures in Paralyzed, Artificially Ventilated Baboons," Archives of Neurology, Vol. 29, No. 2, 1973, pp. 82-87. doi:10.1001/archneur.1973.00490260026003

[10] T. Tomson, U. Lindbom and B. Y. Nilssonm, "Nonconvulsive Status Epilepticus in Adults: Thirty-Two Consecutive Patients from a General Hospital Population," Epilepsi, Vol. 33, No. 5, 1992, pp. 829-835. doi:10.1111/j.1528-1157.1992.tb02190.x

[11] B. Litt, R. J. Wityk, S. H. Hertz, et al., "Nonconvulsive Status Epilepticus in the Critically Ill Elderly," Epilepsia, Vol. 39, No. 11, 1998, pp. 1194-1202. doi:10.1111/j.1528-1157.1998.tb01311.x

[12] F. W. Drislane, "Evidence against Permanent Neurologic Damage from Nonconvulsive Status Epilepticus," Journal of Clinical Neurophysiology, Vol. 16, No. 4, 1999, pp. 323-331. doi:10.1097/00004691-199907000-00004

[13] M. C. Walker, "Diagnosis and Treatment of Nonconvulsive Status Epilepticus," CNS Drugs, Vol. 15, No. 12, 2001, pp. 931-939. doi:10.2165/00023210-200115120-00003

[14] B. F. Shneker and N. B. Fountain, "Assessment of Acute Morbidity and Mortality in Nonconvulsive Status Epilepticus," Neurology, Vol. 61, No. 8, 2003, pp. 1066-1073. 
[15] J. M. Murthy, "Nonconvulsive status epilepticus: An under Diagnosed and Potentially Treatable Condition," Neurology India, Vol. 51, No. 4, 2003, pp. 453-454.

[16] D. M. Treiman, P. D. Meyers, N. Y. Walton, et al., "A Comparison of Four Treatments for Generalized Convulsive status epilepticus. Veterans Affairs Status Epilepticus Cooperative Study Group," New England Journal of Medicine, Vol. 339, 1998, pp. 792-798. doi:10.1056/NEJM199809173391202

[17] R. J. DeLorenzo, E. J. Waterhouse, A. R. Towne, et al., "Persistent Nonconvulsive Status Epilepticus after the Control of Convulsive Status Epilepticus," Epilepsia, Vol. 39, No. 8, 1998, pp. 833-840. doi:10.1111/j.1528-1157.1998.tb01177.x

[18] A. O. Rossetti, S. Hurwitz, G. Logroscino and E. B. Bromfield, "Prognosis of Status Epilepticus: Role of Aetiology, Age, and Consciousness Impairment at Presentation," Journal of Neurology, Neurosurgery \& Psychiatry, Vol. 77, 2006, pp. 611-615. doi:10.1136/jnnp.2005.080887

[19] P. W. Kaplan, "Prognosis in Nonconvulsive Status Epilepticus," Epileptic Disorders, Vol. 2, No. 4, 2000, pp. 185193.

[20] F. J. Bottaro, O. A. Martinez, M. M. Pardal, J. E. Bruetman and R. C. Reisin, "Nonconvulsive Status Epilepticus in the Elderly: A Case-Control Study," Epilepsia, Vol. 48, No. 5, 2007, pp. 966-972. doi:10.1111/j.1528-1167.2007.01033.x

[21] D. H. Lowenstein and M. J. Aminoff, "Clinical and EEG Features of Status Epilepticus in Comatose Patients," Neurology, Vol. 42, No. 1, 1992, pp. 100-104.

[22] F. W. Drislane and D. L. Schomer, "Clinical Implications of Generalized Electrographic Status Epilepticus," Epilepsy Research, Vol. 19, No. 2, 1994, pp. 111-121. doi:10.1016/0920-1211(94)90021-3

[23] J. Scozzafava, M. S. Hussain, P. G. Brindley, M. J. Jacka and D. W. Gross, "The Role of the Standard 20 Minute EEG Recording in the Comatose Patient," Journal of Clinical Neuroscience, Vol. 17, No. 1, 2010, pp. 64-68.

[24] G. B. Young, K. G. Jordan and G. S. Doig, "An Assessment of Nonconvulsive Seizures in the Intensive Care Unit Using Continuous EEG Monitoring: An Investigation of Variables Associated with Mortality," Neurology, Vol. 47, No. 1, 1996, pp. 83-89.

[25] D. J. Chong and L. J. Hirsch, "Which EEG Patterns Warrant Treatment in the Critically Ill? Reviewing the Evidence for Treatment of Periodic Epileptiform Discharges and Related Patterns," Journal of Clinical Neurophysiology, Vol. 22, No. 2, 2005, pp. 79-91. doi:10.1097/01.WNP.0000158699.78529.AF

[26] P. W. Kaplan, "EEG Criteria for Nonconvulsive Status Epilepticus," Epilepsia, Vol. 48, Suppl. 8, 2007, pp. 3941. doi:10.1111/j.1528-1167.2007.01345.x

[27] A. T. Berg, S. F. Berkovic, M. J. Brodie, et al., "Revised Terminology and Concepts for Organization of Seizures and Epilepsies: Report of the ILAE Commission on Classification and Terminology, 2005-2009," Epilepsia, Vol.
51, No. 4, 2010, pp. 676-685.

doi:10.1111/j.1528-1167.2010.02522.x

[28] J. W. Dunne, Q. A. Summers and E. G. Stewart-Wynne, "Non-Convulsive Status Epilepticus: A Prospective Study in an Adult General Hospital," QJM, Vol. 62, No. 2, 1987, pp. 117-126.

[29] M. Siddiqui, N. Jamil, A. Malik, A. Bano, F. S. Khan and K. Siddiqui, "Frequency of Non Convulsive Status Epilepticus in Patients with Impaired Level of Consciousness," Journal of the Pakistan Medical Association, Vol. 59, No. 5, 2009, pp. 296-298.

[30] A. R. Towne, E. J. Waterhouse, J. G. Boggs, et al., "Prevalence of Nonconvulsive Status Epilepticus in Comatose Patients," Neurology, Vol. 54, No. 2, 2000, pp. 340-345.

[31] J. T. Narayanan and J. M. Murthy, "Nonconvulsive Status Epilepticus in a Neurological Intensive Care Unit: Profile in a Developing Country," Epilepsia, Vol. 48, No. 5, 2007, pp. 900-906. doi:10.1111/j.1528-1167.2007.01099.x

[32] M. Privitera, M. Hoffman, J. L. Moore and D. Jester, "EEG Detection of Nontonic-Clonic Status Epilepticus in Patients with Altered Consciousness," Epilepsy Research, Vol. 18, No. 2, 1994, pp. 155-166. doi:10.1016/0920-1211(94)90008-6

[33] G. B. Young and G. S. Doig, "Continuous EEG Monitoring in Comatose Intensive Care Patients: Epileptiform Activity in Etiologically Distinct Groups," Neurocritical Care, Vol. 2, No. 1, 2005, pp. 5-10. doi:10.1385/NCC:2:1:005

[34] R. J. DeLorenzo, W. A. Hauser, A. R. Towne, et al., "A Prospective, Population-Based Epidemiologic Study of Status Epilepticus in Richmond, Virginia," Neurology, Vol. 46, No. 4, 1996, pp. 1029-1035.

[35] F. B. Scholtes, W. O. Renier and H. Meinardi, "NonConvulsive Status Epilepticus: Causes, Treatment, and Outcome in 65 Patients," Journal of Neurology, Neurosurgery \& Psychiatry, Vol. 61, 1996, pp. 93-95. doi:10.1136/jnnp.61.1.93

[36] G. B. Young, J. J. Gilbert and D. W. Zochodne, "The Significance of Myoclonic Status Epilepticus in Postanoxic Coma," Neurology, Vol. 40, 1990, pp. 1843-1848.

[37] A. C. Hui, C. Cheng, A. Lam, V. Mok and G. M. Joynt, "Prognosis Following Postanoxic Myoclonus Status Epilepticus," European Neurology, Vol. 54, No. 1, 2005, pp. 10-13. doi:10.1159/000086755

[38] W. D. Freeman, K. M. Barrett, M. L. Freeman, et al., "Predictors of Awakening from Postanoxic Status Epilepticus after Therapeutic Hypothermia," Neurology, Vol. 73, 2009, pp. 1512-1513. doi:10.1212/WNL.0b013e3181bd6923

[39] A. O. Rossetti, M. Oddo, L. Liaudet and P. W. Kaplan, "Predictors of Awakening from Postanoxic Status Epilepticus after Therapeutic Hypothermia," Neurology, Vol. 72, No. 8, 2009, pp. 744-749. doi:10.1212/01.wnl.0000343006.60851.62

[40] F. Thomke and S. L. Weilemann, "Poor Prognosis Despite Successful Treatment of Postanoxic Generalized Myoclonus," Neurology, Vol. 74, 2010, pp. 1392-1394. 
doi:10.1212/WNL.0b013e3181dad5b9

[41] A. O. Rossetti, G. Logroscino, L. Liaudet, et al., "Status Epilepticus: An Independent Outcome Predictor after Cerebral Anoxia," Neurology, Vol. 69, No. 3, 2007, pp. 255260. doi:10.1212/01.wnl.0000265819.36639.e0

[42] A. O. Rossetti, G. Logroscino and E. B. Bromfield, "Refractory Status Epilepticus: Effect of Treatment Aggressiveness on Prognosis," Archives of Neurology, Vol. 62, 2005, pp. 1698-1702. doi:10.1001/archneur.62.11.1698

[43] A. O. Rossetti, G. Logroscino and E. B. Bromfield, "A Clinical Score for Prognosis of Status Epilepticus in Adults," Neurology, Vol. 66, No. 11, 2006, pp. 1736-1738. doi:10.1212/01.wnl.0000223352.71621.97

[44] P. W. Kaplan, "Nonconvulsive Status Epilepticus," Neurology, Vol. 61, No. 8, 2003, pp. 1035-1036.

[45] F. W. Drislane, M. R. Lopez, A. S. Blum and D. L. Schomer, "Detection and Treatment of Refractory Status Epilepticus in the Intensive Care Unit," Journal of Clini- cal Neurophysiology, Vol. 25, No. 4, 2008, pp. 181-186. doi:10.1097/WNP.0b013e31817be70e

[46] M. A. Granner and S. I. Lee, "Nonconvulsive Status Epilepticus: EEG Analysis in a Large Series," Epilepsia, Vol. 35, No. 1, 1994, pp. 42-47. doi:10.1111/j.1528-1157.1994.tb02910.x

[47] J. Claassen, J. K. Lokin and B. F. Fitzsimmons, F. A. Mendelsohn and S. A. Mayer, "Predictors of Functional Disability and Mortality after Status Epilepticus," Neurology, Vol. 58, 2002, pp. 139-142.

[48] A. Krumholz, G. Y. Sung, R. S. Fisher, E. Barry, G. K. Bergey and L. M. Grattan, "Complex Partial Status Epilepticus Accompanied by Serious Morbidity and Mortality," Neurology, Vol. 45, 1995, pp. 1499-1504.

[49] P. Thomas, A. Beaumanoir, P. Genton, C. Dolisi and M. Chatel, "De Novo Absence Status of Late Onset: Report of 11 Cases," Neurology, Vol. 42, 1992, pp. 104-110. 